\title{
Time-resolved amplified spontaneous emission in quantum dots
}

Cite as: Appl. Phys. Lett. 97, 251106 (2010); https://doi.org/10.1063/1.3529447

Submitted: 26 June 2010 • Accepted: 25 November 2010 • Published Online: 21 December 2010

J. Gomis-Bresco, S. Dommers-Völkel, O. Schöps, et al.

\section{ARTICLES YOU MAY BE INTERESTED IN}

Dynamics of amplified spontaneous emission in InAs/GaAs quantum dots Applied Physics Letters 76, 3507 (2000); https://doi.org/10.1063/1.126689

Multidimensional quantum well laser and temperature dependence of its threshold current Applied Physics Letters 40, 939 (1982); https://doi.org/10.1063/1.92959

From amplified spontaneous emission to microring lasing using nanocrystal quantum dot solids

Applied Physics Letters 81, 1303 (2002); https://doi.org/10.1063/1.1497708

母 QBLOX

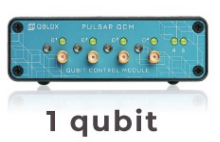

Shorten Setup Time Auto-Calibration More Qubits

Fully-integrated Quantum Control Stacks Ultrastable DC to $18.5 \mathrm{GHz}$ Synchronized $<<1 \mathrm{~ns}$ ultralow noise

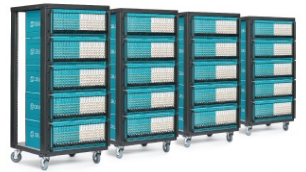

100s qubits

visit our website > 


\title{
Time-resolved amplified spontaneous emission in quantum dots
}

\author{
J. Gomis-Bresco, ${ }^{1, a)}$ S. Dommers-Völkel, ${ }^{2}$ O. Schöps, ${ }^{2}$ Y. Kaptan, ${ }^{2}$ O. Dyatlova, ${ }^{2}$ \\ D. Bimberg, ${ }^{2}$ and U. Woggon ${ }^{2}$ \\ ${ }^{1}$ Institut für Optik und Atomare Physik, Technische Universität Berlin, Strasse des 17, \\ Juni 135, D-10623 Berlin, Germany \\ ${ }^{2}$ Institut für Festkörperphysik, Technische Universität Berlin, Hardenbergstraße 36, \\ D-10623 Berlin, Germany
}

(Received 26 June 2010; accepted 25 November 2010; published online 21 December 2010)

\begin{abstract}
In time-resolved experiments at InGaAs/GaAs quantum-dots-in-a-well (DWELL) semiconductor optical amplifiers, pump-probe of the ground state (GS) population, and complementary measurement of the amplified spontaneous emission of the excited state (ES) population, we are able to separate the early subpicosecond dephasing dynamics from the later picosecond population relaxation dynamics. We observe a 10 ps delay between the nonlinear GS pulse amplification and the subsequent ES population drop-off that supports the dominance of a direct two dimensional reservoir-GS capture relaxation path in electrically pumped quantum-dot-DWELL structures. (C) 2010 American Institute of Physics. [doi:10.1063/1.3529447]
\end{abstract}

Nowadays many optoelectronic devices ${ }^{1,2}$ use quantumdots-in-a-well (DWELL) as active media because they provide faster gain recovery, ${ }^{3}$ lower threshold current, ${ }^{4}$ and pattern free amplification. ${ }^{5}$ An important application of DWELL quantum dots (QDs) is demonstrated in semiconductor optical amplifiers (SOAs), i.e., in an electrically pumped waveguide where the population inversion created in the QDs by the current is used to amplify the propagating light. To investigate the ultrafast optical response of SOA-devices, heterodyne pump and probe experiments became a standard tool to study the effect of doping, ${ }^{6}$ optically induced index of refraction changes, ${ }^{7}$ or the origin and limits of the relaxation of carriers in QDs. ${ }^{3,8-14}$ The understanding and control of the carrier relaxation pathway in DWELL structures are crucial to achieve large operation bandwidths for telecommunications and data applications. In particular, at moderate and high injection currents the dominating relaxation mechanism is Coulomb-scattering in a carrier reservoir. ${ }^{11,15}$ For weak excitation, where carrier-carrier scattering is less efficient, residual populations in the higher quantum-dot states are found and explained in terms of both carrier-phonon and carrier-carrier interactions. ${ }^{16}$

Subpicosecond pump-probe (or other nonlinear-optical) experiments monitor both the population decay and the decoherence process in the QD gain recovery signals within the first picoseconds. Simulations of the QD ground state (GS) gain dynamics by solving the semiconductor QD-Bloch equations of a system composed by the QDs, and a two dimensional (2D)-reservoir (the wetting layer and the quantum well) evidenced that dephasing, nonthermal carrier populations, and Coulomb coupling to the reservoir have to be taken into account, ${ }^{11}$ although we can simplify the Bloch approach and use only a laserlike rate equation model to interpret the experiments after the first picoseconds. ${ }^{12-14}$ In the important case of cumulative amplification of pulse trains, relaxation only through the excited state would produce an ultimate breakdown of the gain recovery ${ }^{17}$ which is not observed in experiments. ${ }^{12}$

${ }^{\text {a)} E l e c t r o n i c ~ m a i l: ~ j o r d i . g o m i s b r e s c o @ t u-b e r l i n . d e . ~}$
In the ongoing discussion of the nature of carrier relaxation pathways we obviously need an alternative experimental method independent of pump-probe experiments and free of coherent effects to test directly the excited state (ES) population after the amplification of a pulse resonant to the QD GS. In this paper, we propose to monitor the timeresolved luminescence of the ES that is not affected by coherent effects but proportional to the instantaneous ES population. We introduce an experimental technique which is based on complementary pump-probe experiments of the ground state population and time-resolved amplified spontaneous emission (TRASE) of the excited state population. This technique is applied to DWELL-SOAs and allows us to observe changes of the ES population within the time resolution of the light detector (6 ps).

The sample studied is a p-i-n structure with a $1 \mathrm{~mm}$ long, $4 \mu \mathrm{m}$ wide waveguide. The active medium consists of 15 layers of molecular beam epitaxy grown QDs-in-a-well with a nominal areal density of $2 \times 10^{10} \mathrm{~cm}^{-2} /$ layer and a nominal delta p-doping of $5 \times 10^{17} \mathrm{~cm}^{-2}$ (for further sample details see Ref. 3). When pumped electrically, we observe a 33 meV broad (inhomogeneous broadening) GS excitonic emission peak at $E_{\mathrm{GS}}=965 \mathrm{meV}(\lambda=1.285 \mu \mathrm{m})$. A well resolved p-shell ES emission peak at $70 \mathrm{meV}$ above the ground state dominated at injection currents above $70 \mathrm{~mA}$.

We used a Ti:sapphire pumped optical parametric oscillator tuned to the GS transition peak, $\lambda=1.285 \mu \mathrm{m}$, corresponding to a photon energy of $965 \mathrm{meV}$ with a pulse width of $150 \mathrm{fs}$ (full width at half maximum) and a repetition rate of $75.4 \mathrm{MHz}$. First we determine in femtosecond-pumpprobe experiment the gain recovery dynamics of the QD GS [Fig. 2(a)]. For the complementary TRASE measurement we coupled the beam into the waveguide and sent the light coupled out to a streak camera. The streak camera, synchronized with the pulsed laser, allowed us to spectrally disperse and time resolve both the amplified pulse and the electroluminescence of the SOAs. Normal spontaneous processes in the SOAs are not time-correlated with the pulsed laser and are statistically averaged. Amplified spontaneous emission (ASE) of the SOA generates a constant background, except for the population changes related to the amplification pro- 


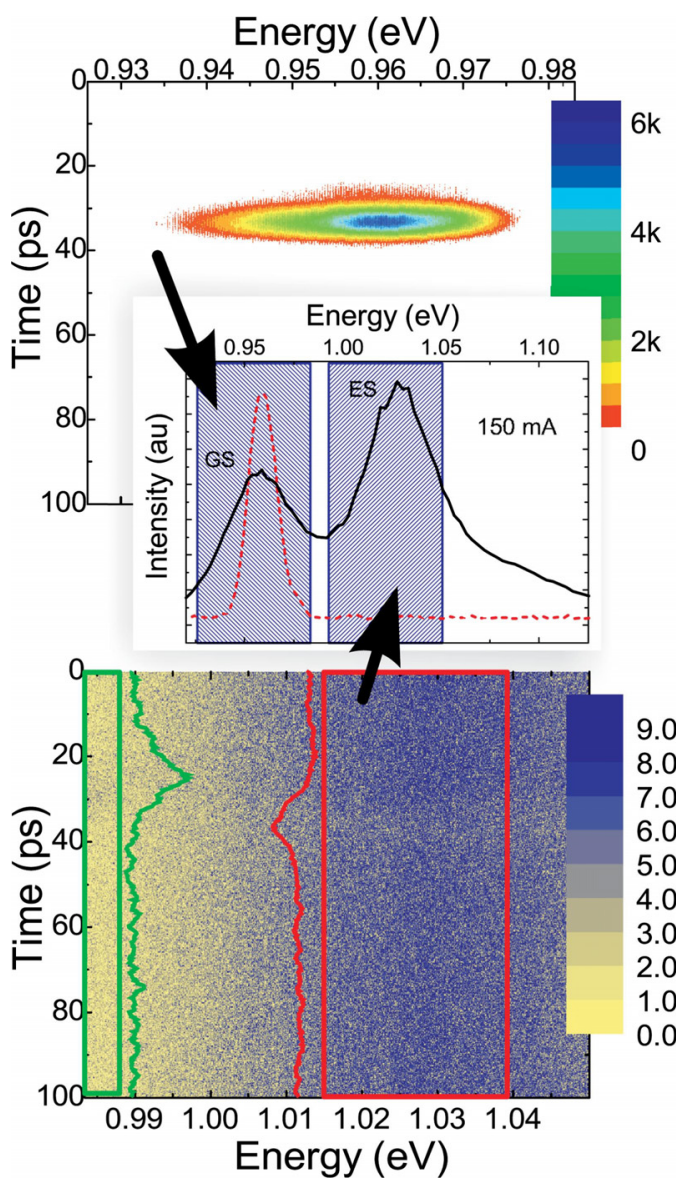

FIG. 1. (Color online) Upper panel: streak camera image of an amplified pulse resonant to the GS. Middle panel: spectra of the ASE at $150 \mathrm{~mA}$ (solid black line) and the pulse coupled in. Left (right) patterned zone marks the spectral range of upper and lower streak images. Lower panel: streak camera image of the amplified spontaneous emission centered at the ES emission and showing the ES ASE (red centered curve) and the signal tail of the pump laser (green left curve).

cess. The low light level of the ASE signal and the time dispersion of the measurement (we measured only $100 \mathrm{ps}$ out of $13 \mathrm{~ns}$ ) resulted in long acquisition times of typically 30 min.

A typical result of the TRASE measurements is shown in Fig. 1: the upper panel is a streak camera image of an amplified pulse measured at injection currents of $150 \mathrm{~mA}$ (limited by the temporal resolution of $6 \mathrm{ps}$ ). Both the ultrafast recovery dynamics of the GS at that injection current ${ }^{3}$ and the pump pulse itself are below the temporal resolution of the streak camera. In the lower panel, we show a streak camera image of the ES spectral window (red centered zone) of the QD DWELL SOA pumped with an injection current of $150 \mathrm{~mA}$ and being in the saturation regime. The spectral integration yields the red centered curve, showing the ES ASE dynamics within the first 100 ps. The arrival time of the laser pulse is marked by the green left curve obtained from spectral integration over the tail of the amplified laser pulse (green left zone). Figure 2 proves that the streak camera images are sensitive to the changes in the ES ASE created by the amplification of the pulse. We observe a pronounced decrease of intensity in the ES TRASE, followed by a partial fast signal recovery up to a value of $16 \%$ of the initial ASE signal lasting then for about 100 ps. The most important result is, however, that the minimum of the ES TRASE ap-

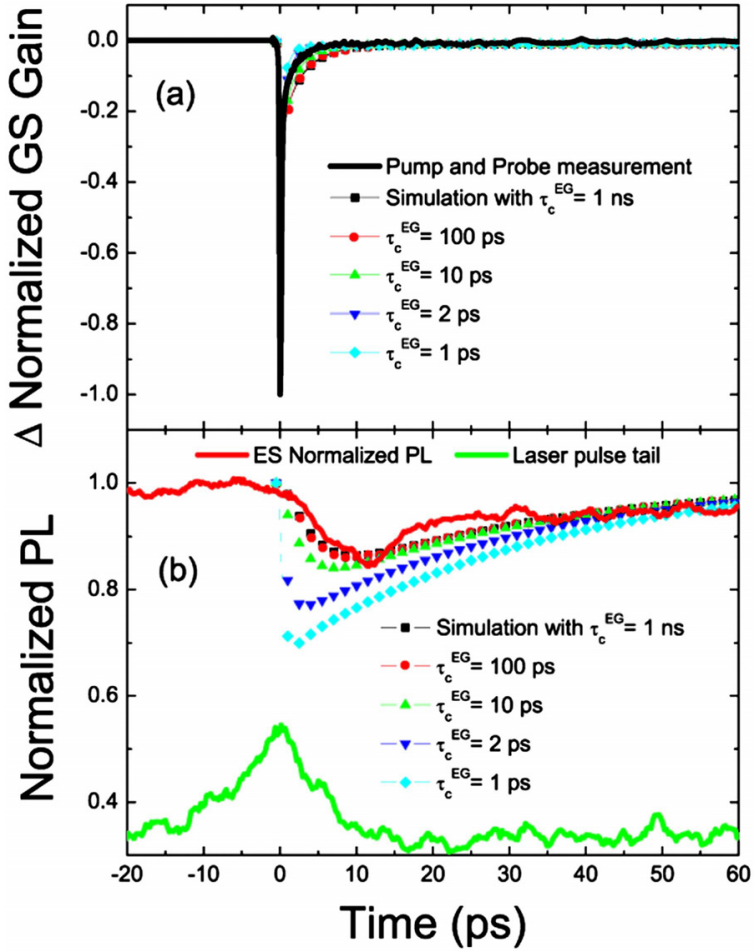

FIG. 2. (Color online) (a) Pump and probe resonant to the GS and (b) TRASE of the ES emission [upper red curve, corresponding to the red centered zone in Fig. 1 lower panel (1015-1039 meV)] and temporal position of the amplified pulse [green lower curve, corresponding to the laser tail seen in the green left zone in Fig. 1 lower panel (983-988 meV)]. We plot simulations of the expected saturation behavior as a function of the intradot relaxation time for (a) GS and (b) ES.

pears $10 \mathrm{ps}$ after the GS laser pulse amplification. A hypothetical fast relaxation from the ES to the GS, accompanied by the slower gain recovery of the ES (see Refs. 3, 18, and 22 ), would produce a fast decrease of the ES population which is not observed in the TRASE experiment of Fig. 2 or any other measurement with less pump power. To explain the observed ASE dynamics in TRASE experiments, we apply a population model and compare the signatures of the different carrier relaxation paths with the experimental data. The developed rate equation system is given by

$$
\begin{aligned}
\frac{\partial N_{B}}{\partial t}= & J-\frac{N_{B}}{\tau_{c}^{W B}}\left(1-\frac{N_{W}}{N_{W}^{\max }}\right)+\frac{N_{W}}{\tau_{e}^{B W}}-\frac{N_{B}}{\tau_{s p}^{B}}, \\
\frac{\partial N_{W}}{\partial t}= & \frac{N_{B}}{\tau_{c}^{W B}}\left(1-\frac{N_{W}}{N_{W}^{\max }}\right)-\frac{N_{W}}{\tau_{e}^{B W}}-\frac{N_{W}}{\tau_{c}^{W G}}\left(1-N_{G}\right) \\
& +\frac{N_{E}}{\tau_{e}^{E W}}\left(1-\frac{N_{W}}{N_{W}^{\max }}\right)+\frac{N_{W}}{\tau_{c}^{W E}}\left(1-\frac{N_{E}}{2}\right) \\
& +\frac{N_{G}}{\tau_{e}^{G W}}\left(1-\frac{N_{W}}{N_{W}^{\max }}\right)-\frac{N_{W}}{\tau_{s p}^{W}}
\end{aligned}
$$

$$
\begin{aligned}
\frac{\partial N_{E}}{\partial t}= & \frac{N_{W}}{\tau_{c}^{W E}}\left(1-\frac{N_{E}}{2}\right)-\frac{N_{E}}{\tau_{e}^{E W}}\left(1-\frac{N_{W}}{N_{W}^{\max }}\right)+\frac{N_{G}}{\tau_{e}^{G E}}\left(1-\frac{N_{E}}{2}\right) \\
& -\frac{N_{E}}{\tau_{c}^{E G}}\left(1-N_{G}\right)-\frac{N_{E}}{\tau_{s p}}
\end{aligned}
$$




$$
\begin{aligned}
\frac{\partial N_{G}}{\partial t}= & \frac{N_{E}}{\tau_{c}^{E G}}\left(1-N_{G}\right)+\frac{N_{W}}{\tau_{c}^{W G}}\left(1-N_{G}\right)-\frac{N_{G}}{\tau_{s p}} \\
& -\frac{N_{G}}{\tau_{e}^{G W}}\left(1-\frac{N_{W}}{N_{W}^{\max }}\right)-\frac{N_{G}}{\tau_{e}^{G E}}\left(1-\frac{N_{E}}{2}\right) \\
& -g P\left(N_{G}-\frac{1}{2}\right) .
\end{aligned}
$$

We model the experimental data by a four-level system including the carrier population of the QD ground $\left(N_{G}\right)$ and excited $\left(N_{E}\right)$ states, the 2D-reservoir (quantum well and wetting layer $\left.N_{W}\right)$, and the GaAs barrier $\left(N_{B}\right)$. We include as parameters the capture times $\tau_{c}^{\text {OriginDestiny }} . P$, the uncoupled light power, is chosen to satisfy the saturation regime. The escape times are calculated as $\tau_{e}^{\text {OriginDestiny }}$ $=\tau_{c}^{\text {DestinyOrigin }} \exp ^{E_{\text {Destiny }}-E_{\text {Origin }} / k T}$. The other parameters used are $E_{G}=0 \mathrm{meV}, E_{E}=60 \mathrm{meV}, E_{W}=120 \mathrm{meV}, E_{B}$ $=130 \mathrm{meV}, \tau_{C}^{W E}=\tau_{C}^{W G}=1 \mathrm{ps}, \tau_{s p}=400 \mathrm{ps}, \tau_{s p}^{W}=20 \mathrm{ps}, \tau_{s p}^{B}$ $=10 \mathrm{ps}, \quad \tau_{C}^{B W}=300 \mathrm{ps}, \quad g=10, \quad N_{W}^{\max }=20$ carriers, and $J$ $=1.4$ carriers $/$ ps. The carrier/relaxation rates depend on the carrier population, but for a fixed injection current, we consider them constant.

Supposing that direct capture and cascadelike capture are both possible, we plot in Fig. 2 the simulations for the time evolution of the GS (a) and ES (b) populations as a function of the intradot relaxation time $\tau_{c}^{E G}$, but fixing the capture times $\tau_{c}^{W E}$ and $\tau_{c}^{W G}$ to $1 \mathrm{ps}$, which is mandatory to reproduce the ultrafast ground state population recovery found in literature. We show in Fig. 2(a) a standard one color ground state pump and probe at the same experimental conditions used in Figs. 1 and 2(b): the GS population recovers fully in $5 \mathrm{ps}$, feature that is well reproduced by the simulations. $\tau_{C}^{E G}$ is the modified parameter starting from $\tau_{C}^{E G}$ $=1000$ ps, i.e., almost completely switching off the intradot, cascadelike relaxation pathway, and varied then down to $\tau_{C}^{E G}=100,10,2$, and 1 ps intradot carrier relaxation times. The delay found is not compatible with a cascadelike relaxation path because in that case, the ES TRASE decrease should appear almost immediately after pulse amplification. Good agreement of the simulations and the experimental results is obtained for an intradot relaxation of $\tau_{C}^{E G}>10 \mathrm{ps}$, which is 10 times slower than the direct capture time $\tau_{C}^{W G}$. Our results contrast with the evidences of cascadelike capture found in experiments with much lower excitation density. ${ }^{19,20}$ Coulomb-scattering is likely to be responsible for our fast carrier capture and relaxation: the relaxation path depends on the carrier density. ${ }^{21}$ It should be mentioned here that the observed early picosecond ES ASE dynamics in TRASE experiments differ from the ES-GS pump and probe results, as, e.g., observed in Ref. 22, confirming the absence of coherent effects in ASE signals.
In conclusion, in complementary pump-probe experiments of the GS population and TRASE measurement of the ES population we directly determined the existence of a direct capture relaxation path in a quantum-dot-in-a-well semiconductor optical amplifier. We base our conclusion on the delayed ES population response observed in the saturation regime and the lack of ultrafast response at lower pump powers that are incompatible with a dominance of a cascade like relaxation path.

We acknowledge the funding of this work by Grant No. SFB 787 of the DFG.

${ }^{1}$ G. Fiol, D. Arsenijevic, D. Bimberg, A. G. Vladimirov, M. Wolfrum, E. A. Viktorov, and P. Mandel, Appl. Phys. Lett. 96, 011104 (2010).

${ }^{2}$ C. Meuer, J. Kim, M. Laemmlin, S. Liebich, G. Eisenstein, R. Bonk, T. Vallaitis, J. Leuthold, A. Kovsh, I. Krestnikov, and D. Bimberg, IEEE J. Sel. Top. Quantum Electron. 15, 749 (2009).

${ }^{3}$ S. Dommers, V. V. Temnov, U. Woggon, J. Gomis, J. Martinez-Pastor, M. Laemmlin, and D. Bimberg, Appl. Phys. Lett. 90, 033508 (2007).

${ }^{4}$ D. Bimberg, G. Fiol, M. Kuntz, C. Meuer, M. Laemmlin, and N. N. Ledentsov, Phys. Status Solidi A 203, 3523 (2006).

${ }^{5}$ T. Akiyama, N. Hatori, Y. Nakata, H. Ebe, and M. Sugawara, Electron. Lett. 38, 1139 (2002)

${ }^{6}$ V. Cesari, W. Langbein, P. Borri, M. Rossetti, A. Fiore, S. Mikhrin, I. Krestnikov, and A. Kovsh, Appl. Phys. Lett. 90, 201103 (2007).

${ }^{7}$ I. O’Driscoll, T. Piwonski, J. Houlihan, G. Huyet, R. J. Manning, and B. Corbett, Appl. Phys. Lett. 91, 263506 (2007).

${ }^{8}$ P. Borri, F. Romstad, W. Langbein, A. Kelly, J. Mork, and J. Hvam, Opt. Express 7, 107 (2000).

${ }^{9}$ M. Ishida, N. Hatori, T. Akiyama, K. Otsubo, Y. Nakata, H. Ebe, M. Sugawara, and Y. Arakawa, Appl. Phys. Lett. 85, 4145 (2004).

${ }^{10}$ T. Piwonski, I. O'Driscoll, J. Houlihan, G. Huyet, R. J. Manning, and A. V. Uskov, Appl. Phys. Lett. 90, 122108 (2007).

${ }^{11}$ J. Gomis-Bresco, S. Dommers, V. V. Temnov, U. Woggon, M. Laemmlin, D. Bimberg, E. Malic, M. Richter, E. Schöll, and A. Knorr, Phys. Rev. Lett. 101, 256803 (2008)

${ }^{12}$ J. Gomis-Bresco, S. Dommers, V. Temnov, U. Woggon, J. MartinezPastor, M. Laemmlin, and D. Bimberg, IEEE J. Quantum Electron. 45, 1121 (2009).

${ }^{13}$ J. Kim, C. Meuer, D. Bimberg, and G. Eisenstein, Appl. Phys. Lett. 94, 041112 (2009).

${ }^{14}$ T. Erneux, E. A. Viktorov, P. Mandel, T. Piwonski, G. Huyet, and J. Houlihan, Appl. Phys. Lett. 94, 113501 (2009).

${ }^{15}$ R. P. Prasankumar, W. W. Chow, J. Urayama, R. S. Attaluri, R. V. Shenoi, S. Krishna, and A. J. Taylor, Appl. Phys. Lett. 96, 031110 (2010).

${ }^{16}$ H. Kurtze, J. Seebeck, P. Gartner, D. R. Yakovlev, D. Reuter, A. D. Wieck, M. Bayer, and F. Jahnke, Phys. Rev. B 80, 235319 (2009).

${ }^{17}$ T. Berg, S. Bischoff, I. Magnusdottir, and J. Mork, IEEE Photonics Technol. Lett. 13, 541 (2001).

${ }^{18}$ S. Schneider, P. Borri, W. Langbein, U. Woggon, R. Sellin, D. Ouyang, and D. Bimberg, IEEE Photonics Technol. Lett. 17, 2014 (2005).

${ }^{19}$ T. Müller, F. F. Schrey, G. Strasser, and K. Unterrainer, Appl. Phys. Lett. 83, 3572 (2003).

${ }^{20}$ T. Siegert, S. Marcinkevicius, and Q. X. Zhao, Phys. Rev. B 72, 085316 (2005).

${ }^{21}$ B. Ohnesorge, M. Albrecht, J. Oshinowo, A. Forchel, and Y. Arakawa, Phys. Rev. B 54, 11532 (1996).

${ }^{22}$ I. O'Driscoll, T. Piwonski, C.-F. Schleussner, J. Houlihan, G. Huyet, and R. J. Manning, Appl. Phys. Lett. 91, 071111 (2007). 